\title{
Moral Communication and Moral Values in the Moral Dilemma About Martha and Mary
}

\author{
Victorita Trif \\ Faculty of Psychology and Educational Sciences, University of Bucharest, Bucharest, Romania \\ Email address: \\ victoriatrif@yahoo.com

\section{To cite this article:} \\ Victorita Trif. Moral Communication and Moral Values in the Moral Dilemma About Martha and Mary. Education Journal. Special Issue: \\ Education and Moral Values: Authenticity, Countercultures and Standardization or Ethics - A New Chernobyl? Vol. 9, No. 3, 2020 , pp. 80-88. \\ doi: $10.11648 /$ j.edu.20200903.14
}

Received: May 15, 2020; Accepted: June 2, 2020; Published: June 20, 2020

\begin{abstract}
This article aims to investigate the integrity taking into account the relationship between culture and psychology. The conceptual framework is very sophisticated because of the wide range of studies from philosophy, education, psychology, communication, linguistic and semiotics, neurosciences, law, and so on. The multiplicity of efforts to design meta-ethics and the international needs to assume some principles and guidelines reveal the importance of the approach. The text from the Bible about Martha and Mary - a moral dilemma - becomes the start point of the investigation. The text could be considered a story, a case-study in terms of meta-ethics, a problem-solving or a hypothesis to examine moral brain. The qualitative analysis of the findings offer - despite the difficulty of moral reasoning - academically substantial inferences regarding legal decisions. For example, each judicial case could use in ethical reasoning, the similar routes of moral reasoning. The target population (660 students) was invited to critically assess the position of Martha and the position of Mary, then to explain their moral judgment behind the answer. The application stimulates moral thinking and critical thinking. To conclude, moral cognition is related to moral communication. There are various advantages for empirical work taking into account the transferability and the generalizability of findings. It is important that the formula of moral communication pretext can be adapted to suit a wide variety of research situations and various purposes.
\end{abstract}

Keywords: Moral Communication, Moral Values, Honesty, Moral Dilemma, Moral Education, Moral Paradigm

\section{Introduction}

In the contemporary crisis the culture of dishonesty growing up, the social recognition of the honesty is deteriorated and the implications for the ethical behavior in day by day life are problematic [1]. The meta-analysis of the conceptual framework reveals a wide variation across academic trends and conventions [2], best practices and practical orientations as well as psychosocial assumptions. Meta-cultural relativists, teachers, managers, bioethicists, magistrates, clerical workers, etc. considers honesty a common denominator for effective communications about values. There are various types of knowledge on moral dimension of life from the vantage point of different perspectives: theoretical, experimental, cultural, hypothesized in terms of moral intelligence, spiritual intelligence, existential intelligence, moral law, moral brain, critical thinking and moral reasoning, moral development, observational learning, moral rights, moral communication, social justice, moral values, neuro-ethics, emotive ethics, bioethics, moral conscience, dilemmas, and so on. This means that there are multiple debates about performance management, ethical behavior, metrics of company culture (e. g. organization's values), skills assessment, high ethical standards, workforce integrity, codes of ethics, etc. On the other hand, under the coverage of Utilitarianism and Kantianism or other different moral perspectives integrated in scientific knowledge, the mechanisms involved in processing integrity are ones of the most complex and controversial of all topics. The experimental design, philosophical wording, multicultural perspectives, computational exploration, semiotics analysis exemplify a multiplicity of ways that reframe the moral case deliberation. The psychological investigations, religious approaches, genomic and metagenomic perspectives, ethnographic researches, potential reflective interplay, all produce multidisciplinary approaches 
in order to measure the ethical communication. For ones, investigating integrity could be considered a waste of time; for others, is the key sense of life because it is strongly related to measuring attitudes towards dishonest behavior. Currently, conscientiousness, trustworthy and dependable is correlated with benchmark integrity. For all is a way to reduce human risk or a multidisciplinary paradigm of universe. The meta-analysis of the formal rhetoric legitimizes the huge concern of moral cognition. The recent development in researching integrity include a great number of publications on the topic that contain various key terms of ethics academic market: moral brain, moral cognition, moral reasoning, moral judgment, moral development, moral values, moral communication, moral conscience, ethics, moral law, just memory, dignity, obedience, respect, etc. A diversity of research methodologies, different tools and various discourses are used. Rapid changes in society at a global scale are causing new circumstances where people with integrity leads to authentic behavior. The basic remarks state that the results are often irreconcilables: the findings provide various versions of the reality, counterfeit, corruption, dishonest daily practices, mistakes in moral reasoning desubstantiate the transparent (formal) guidelines. The comparative analysis of the studies provides that some questions are important for delineating alternatives to present technical investigations: why (aims)?, what (results)?, when (times)?, who (intellectual patterns of the assessors)?, where (context or culture, local or global)?, how (research paradigms)?

\section{Research Methodology}

This paper is concerned with various forms of communication around the relationship between culture, psychology and current moral issues. The global crisis generated by COVID-19 is related to the moral decision making and leadership because of the limits to manipulation of human life from genetics and of the limits to the manipulation of medical treatment as well as of living nature. This argues the plethora of challenges exacerbating values crisis that is reflected in the mental about integrity (e. g. minimal positive morality versus minimal negative morality, virtues versus vices, the pressure for safety and the social ethos or pressure to returning at the everyday life, democracy versus global crisis).

The reflexive approach is sustained by spiritual intelligence [3] and polarized paradigms [4]. In practical terms, this research could be considered an innovation exploring the psychological camp of integrity by an unconventional approach. In an action research, the target population was asked about their moral reasoning, starting by a Gospel. The importance of the approach is to illustrate the psychological features of the integrity by culture dependence. The story about Mary and Martha in practical- applicative plan becomes a tool comparable with the techniques to measure honesty in the magistracy culture. The investigation is based on the consideration of M. Kaptein (2005) considerations on instruments design [5]. The analysis of assessment practices for testing the candidates to the magistracy profession ('calling card', 'interview test', 'observations grid') required in United Kingdom, Germany, France, Australia, reveals the needs of collecting 'strong facts' on moral reasoning of the respondents [6]. Starting from this issue the investigation frames the structure of the interview, having the following objectives: to identify moral communication ability, to examine personal philosophy of life of the respondent and to investigate self-evaluation of the respondent. Because of the high level of research sophistication and based on studies addressing moral reasoning, the paper discusses an important part of this effort, one of the hypothesis:

Moral cognition is related to moral communication.

This assumption is considered convergent with potential determinants of moral reasoning. The article aims to examine the moral communication. Because of the vulnerabilities of social context from Romania, as from all the countries in transition, the participants were asked, starting by a stimulus selected from Christian theology:

"Now it came to pass, as they went, that he entered into a certain village: and a certain women named Martha received him into her house. And she had a sister called Mary, which also sat at Jesus' feet and heard his word. But Martha was cumbered about much serving, and came to him, and said, Lord, dost thou not care that my sister hath left me to serve alone? Bid her therefore that she help me. And Jesus answered and said unto her, Martha, Martha, thou art careful and troubled about many things. But one thing is needful: and Mary hath chosen that good part, which shall not be taken away from her."

The danger of contaminating the data from the vantage point of ideologies or political premises is avoided. The target population was asked to comment the text from the Bible regarding the ethics of Martha and the ethics of Mary. The investigation was conducted in the university in the period 2011-2016. Of 660 investigated participants, 630 answered. The target population consists in students in the Service Teachers Training and in Teachers Training, in their first and second academic year, males and females, aged 20 to 25 . The students were invited to assess critically the position of Martha and the position of Mary and to explain their moral judgment behind the answer. The longitudinal investigation was conducted in the following phases:

1. the hypothetical phase (the meanings of the honesty within Romanian axiology space were explored) (20112012),

2. the confirmatory phase (the validity and the reliability of the methods were confirmed) (2012-2013),

3 . the phase of qualitative research (the instrument was applied) (2013-2020).

The narratives were encoded in terms of integrity metrics according to Kaptein's standardized tool to measure honesty [5]. The multi-level knowledge on research moral cognition varies depending on the paradigm: ethnomethodology, semiotic and linguistics, symbolic, structural, psychology, 
comparative cognition, etc. Even the research methodology is qualitative, the theoretical base of the investigation clearly introduces objective parameters of measurement: validity, reliability, consistency, realistic expectations, demanding, common criteria, give consequential appreciations. The convention used in this research is that of defining integrity as a moral principle to guide life. According to the conceptual frame, the personal potential of the respondents (skills, interests, values, temperament factors, external factors) is convergent with the action research. The researcher and the researched co-create data, action research becomes action science and the context of their social world is part of the knowledge. It is normal to regard instrumenting phenomena from this investigation by a reflexive manner. Thus, culturally meaningful data, as a result of the application, are included. The features of the selected discourse consist in multimodality: simultaneously connects the target population to various social contexts, wonder concerning normative premises of day by day life, advance cultural pluralism, combine both internal and external paradigms of the research [4], contrast with conventional / formal debates on moral issues, cross borders from local to global ethics.

\section{Findings}

The findings offer rich sources for full understandings of ethical issues. The psychological bases of heuristics are presented as part of natural settings: each person in our contemporary societies makes moral decisions. The personal values and the imposed ethical rules are sometime adversaries. The moral dilemma becomes a tool to investigate moral behavior. The respondents were encouraged to detach personal feelings from legal Romanian law and to balance between Martha's and Mary's position. Their individual moral decision-making [7] was collected in written form. The Gospel is considered a case method in terms of judicial research, a case-study or a structured interview from the vantage point of research methodology and a simulated situation in terms of law school. The consequence of this approach is to delineate the relevant features of such sophisticated particular case. The identities that the target population may adopt conduct the factual investigation from moral to legal law. Beyond the dissimilarities generated by the discourses on integrity, the text as stimulus incorporates ethical, legal and social problems. The Christian theology in this case argues the problematic framework on moral judgment. From the vantage point of analytic coding, the data collected from the longitudinal research is due to following analysis categories:

1. moral thinking is related to moral conscience;

2. moral thinking is a result of routinized connections from the brain;

3. moral decision making is a result of comparative analysis;

4. subjective moral reasoning is generated by conflict and plurality values;

5. cognitive styles are related to moral brain;
6. it is a multi-dimensional account of moral thinking.

The concepts delineated in the categories of analysis describe the continuum of abstraction from a more specific (concrete) to a more abstract (general) level that is, paradoxically, linked twofold - in qualitative and in quantitative explanations. Comparison is fundamental but the purposes are encapsulated in qualitative framework; thus the findings are centrally concerned this meaning [2]. The qualitative analysis engages the respondents in the problem of justice in our contemporary multicultural societies. Even if the investigation was conducted in Romania, the reflections on ethics are cross-cultural. The Gospel about Martha and Mary is a complex stimulus that can incite to reconsider questioning values. In that sense, there are many values: integrity, self-respect, self-discipline, trust, love, commitment, diversity, care (for others), respect (for others), etc. The judgment is made by a key question involved deliberately in the analysis: Which of the decision is the moral one, the decision of Martha or the decision of Mary? Accepting the explicit formulation from the data, the empirical findings take into account the six classified perspectives questioning answers rather than answering questions. The whole process of how the decision- making is done demonstrates that there are many causes, consequences, experiences integrated in answers.

For most of the respondents, moral thinking indicates moral consciousness. Communication on moral consciousness requires to evaluate probabilities and to deliberate on risks. Since the potential risk is divided between twofold values, the narratives become substantial because evidence two opposes actions: Martha's justification and the decision of Mary. In essence, it is epistemologically risky to deliberate and to explore the alternatives as rational probability. Greater doubt arises from the self-contradicting rules of moral principles. It is clear that the decision-making, as a procedure to norm real-life situations split the moral cognition continuum in errors or cognitive mistakes (low or imprecise probability to choose) and fair example (high probability of rational choice). The rate of admitting the misconducting and the good way among the respondents is $90 \%$. Perhaps the feeling of moral uncertainty, involved within this exploratory Gospel, might affect the mental model of decision-making. The assessment probability is ambiguous as a result of the possible conflicts between personal values and moral values, identified in both moral models. The respondents reported that the probability of misconduct is perceived as huge:"when it was imperative to choose appeared moral consciousness" (57; 58; 63; 64). In regard to the differences between qualitative answers compared to statistical metrics from the fieldwork, these are not significant. Another notable result was the clear pattern of moral dimension; the wording is very consistent concerning high honesty: "-When I have to choose between Martha and Mary, often I tend to give reason to Martha. Simultaneously activates my conscience that is the voice of the God in men. I try to play Martha's and Mary's roles and to identify my view point" (47). From the psychological perspective, the 
dilemmas treated interpersonal conflicts comparable with daily life people; the moral social functions are in relationships with people's limits of moral reasoning. The moral thinking, involved in the meaning of the Gospel, doesn't conduct the respondents by expected rules or by a written version of the good answer. In this research, the target population was asked to interpret the task as a moral solution to a problem. From the perspective of psychological constraints of the dilemmas, the investigation intends to probe the moral judgment. The findings prove that the participants were in trouble to defend themselves against the possibility to have consequences - the danger to score low stages of honesty is evoked significantly in the collected narratives. As an example, a student who achieves a high level of integrity processes an ideal solution: "I am grieved that I have not reached the status of Mary" (27). There was an indicator of the cognitive representation about interpersonal conflicts involved in the item in terms of situational variation. In addition to these, the complexity of moral measurement is demonstrated by the individual strategies used to discover the most moral solution. $92 \%$ of them explained that they are playing complementary roles in order to avoid moral errors. Most of the students postulated that the moral judgment is more cognitively sophisticate and recognized the roles of the social affective and cognitive processes in decision making. It has been argued that the mental structures of moral dilemmas are based on moral conscience: The moral conscience makes me choose Mary" $(1,4,5)$. From the vantage point of social reasoning, the moral conscience is considered the highest level of person's maturity; from the perspective of neuroscience, the investigation examine the moral judgment maturity at neuromaturation level (moral conscience is the sign of the anatomical and chemical characteristics of the brain); in terms of psychology, the moral conscience is linked to personality. The assertion that the moral development is connected to social cognition and social emotion implies to discuss the data from the perspectives of social sensitivity, empathy, intentionality.

The following answer indicates that the moral scenarios proposed are consistent: "The moral conscience dictated me what to do. I thought about what Christ would do. The moral conscience determined me to choose Mary"(16). In contrast to the past-communist ideologies implicated in daily experiences from Romania, the dilemmas from the Gospel generate 'impersonal' moral reasoning. Education is perceived as inculcated values: "The chosen model - Mary's ethics - reinforces my moral and religious conscience" (38). The lived experiences can give a subjective, interpretive and scientific thinking in terms of everyday interactions: "from the vantage point of theology, Mary fulfilled the God's law" (35).

Moral thinking is dependent from the brain' routinized connections. Moral judgment is taken from "learning of parables or dilemmas whose solutions were previously learned" (20) or from the pre-existing knowledge of the nature of moral (4). The meanings, the structure and the interactional context are parts of moral and social representations:"The pre-existent knowledge ... the thought about which of the commandments of the Lord must be respected. A very likely mad me ballance between two commandments of the Lord... Basically by both commands we serve the Lord; hence the difficulty of deciding which model to offer high above" $(8,11,12)$. The narrator's point of view provides the interest in structural brain connectivity and moral learning: "Perhaps my brain mad connections with what I have lived, what defines or characterizes me in order to make a decision" (25). Using narratives can explore the brain mechanisms in order to give details on micro-, mesoand macroscale brain network: "When I have to choose between Martha and Mary, my mind makes connection with other similar dilemmas... I made connections with other parables from the Bible" (28).

Moral rationale is based on comparative analysis: "When I have to choose between Martha and Mary I think that, in fact, I must take the best from each" (3). Examples express rules of subjective moral reasoning generated by valuing personal choice. The target population is thinking of themselves; action research was essential for picturing the personal identity. The human psychological activities are problematic in sense of revealing the neurobiological mechanisms. In this case, the problem solving from the Gospel contributes in offering consistent data, in order to explore the variability of people's moral reasoning. It seems controversial, but the construal level of personal moral judgment is different from the self-construed personality often reported in the literature: "In fact, the attitude of Mary and the attitude of Martha are both subjective. We can-not categorize the good one and the bad one. It is very important here the discernment capacity" (6). The question about values might be posed at different levels. As axiology, the theological level, deontological level and consequentialist level are distinguished. On the other hand, the investigation context consists in repudiation of the personal accepted values. This level of research goes beyond formal interviews requiring more than simply an opinion. The purpose of the study is to identify patterns of moral judgment associated with values crises having application both in practical knowledge and skills. From the vantage point of lifestyles, Mary and Martha exemplify the microcosms of the society. Moral distress is expressed in adjectival and adverbial forms of the research objectivity as in notably empirical data: "Formally, I chose Mary ethics. I am heading to Martha; the Lord says clear ..." (31). The paragraph selected could argue that the strong objectivity belongs to internal and external life of the respondent.

The cognitive styles are related to moral brain; for example, properties of the brain are indicated in a convenient way. The moral brain works by analyzing differences: "In my brain there is a distinction between being spiritual and work ... It is preferable to become Mary at the beiginning, but it is important become Martha ...starring Christ in us" (7) "Initially I didn't chose, I analyzed both behaviors (12). The neural communication in the brain is illustrated in language: "It was a dilemma both - Martha and Mary - are doing 
essentials" (13). The structural parameters of cortical areas are themes included in the microscopic analysis of self: "Both perspectives should be well-analysed... I reported the evangelical pericope to contemporary society." (43). The cortical cytoarchitecture is transcribed in narrative: "I analysed both situations" (62).

The analysis of data collected proves that the 'good thinking' correlates with 'honesty': “I was thinking rigurously about decision-making" (9); "I was in doubt... I wonder at myself and I doubt about decision-making" (10). The ethical conduct reflects the need of moral principles. The rhetoric is not a 'false' production of discourses, there is not an artificial or a fail statement. The criticism of the answers encodes a humanistic way to choose the right way: "in other words it is a battle between arguments of which the winner is decision-making based on Lord' words ... the moral law" (15). Within the makers of the narratives are merely good analysts: "There is a fight: what to do?" (18) Unsurprisingly, the work realistically involves very complex psychological resorts: “... it was difficult to chose; Martha and Mary have done the same" (19). There are a lot of personal benchmarks and indicators drafting the moral reasoning - beliefs, knowledge, attitudes, values, official agreements, conventions, expectations, life rules, cultural principles, etc. The duality is largely assumed: "I don't know if it was right to choose Martha or Mary" (22). As one respondent comment, the skills involved are in conflict: "the problem related in the Gospel is the dispute between two moral principles. From my view point, the principles complete each other. Several times I follow the example of Mary... I try to choose the best part / good part... I would not have chosen" (29). The moral language is expressed in terms of cognitive moral representations about integrity: 'moral norms', 'moral law', 'dilemmas', 'moral rights', 'obligations', 'two ethics'. The moral thinking is connected to emotion and social context: 'tension', 'battle', 'war', 'social normativity', etc. The moral space is pictured as moral rules translated by a linguistic apparatus: a 'moral problem', a 'problem-solving', 'twofold ethics', 'polarized principles', 'two routes/ways', 'two minds'. The terms reveal prototypes based on cognitive constructs. The moral thoughts are essentials for moral reasoning, the meta-ethics issues are stated in atypical answers: "I choose Mary because of my theological education; otherwise I would be a good host" (61). There are many multifaceted meanings and multi-dimensional account of moral thinking.

It has been proposed maintaining alongside the investigation the obligations of the impersonal reason objectivity, empiricism and truth. This approach is linked conceptually to reflexive anthropology, because it conveys cultural meanings about integrity. The dilemma could be considered a tool to measure honesty as accurately and efficiently as possible. The research contribute to missing important elements from our life: multiple types of thoughts, confronting multifaceted social situations, various goals, diverse judgements, associations, brain mechanisms, internal metrics of values, etc.

\section{Discussion}

Originally, the Gospel incorporates the universal thesis of connections between personality, responsibility and social practices. This is a paradox: the complex network may be subject to concrete and abstract meanings. Concretely, the story presents two women having the same status, same legal duties, same legal and moral rights and obligations. The abstract elements surprise by the different regime of taxation (or attribution), "the good part". The difficulties come from the implications of moral sanctions: are two personalities with conscious minds and normal behaviors. The assessment of their responsibility must be just and equitable. In order to understand moral judgment, it is important the psychologic context: one of them wants to be punished and her mental as ,victim' will be affected less or more. Logically, it is necessary to assess the personalities from the vantage point of moral responsibility. Intentionality or culpability, two concepts active in the classic theory about moral development, are not able to solve the moral problem from the Gospel. There are other features of judicial law-making process: the necessity of moral choice. Beyond the paradox, the choice between "the good part" and another "part" has considerable symbolic and politic importance in our contemporary societies:

1. How could citizens be protected from the coercive power of the state?

2. Does the Gospel advertise against the public law illegality?

As case-study, the story is very controversial [4]: its degree of abstractness and universality could be understood in various contexts. It would explain the styles of distributive justice - what it means to be responsible and what are our responsibilities. Perhaps it is impossible to ignore that there are adverse outcomes in the fieldwork expressed by the American realism, the English references, in the light of modernism, post-modernism, post-structuralism, viewed in the ordinary society or in the intellectual thinking. The question of Martha illustrates a conversational meaning and a presupposition. As context-independent knowledge, the semantic or literal meaning can't be contradicted. The defects and the dangers of interrogation as behavior came from the contextual interpretation of the issue. Uncertainty appears in terms of normative judgments. Social effects of moral disagreement are engaged in the question: " Lord, dost thou not care that my sister hath left me to serve alone?" As an intended meaning, it may be about the practical moral reasoning; as communicative or asserted the content is an exemplification of mixing literal meaning, contextual meaning, real conceptual meaning and interpreted meaning. The vocabulary is familiar, the central aim is well delineated, the speakers receive and send concerns, values and norms. These originate the shift of the discussion from moral cognition to moral brain. In this turn-taking, it is possible that the different types of neuronal activities (dilemma as a task) to determine significant mechanisms in the brain registers. May be that the Gospel presents two minds as functions of 
two different moral brains - moral duties and obligations and social duties and obligations which tells us about moral risks. In law wording, the responsibility - as concept and practice refers to public law and particular law. Beyond the theoretical literature, the telling repairs the discrimination because the context is both public and particular. The mental experiences on ethics from the wide world are considered mutually exclusive; the integrity as a principle is widely shared from the citizen' mind to official and un-official law. Integrity is experienced in a variety of ways or not?

The findings demonstrate that there are not limitations from the vantage point of adverse effects (language, political mixture, ethnicity, race). The verbal discourses are not influenced explicitly by the language of the press concerning social justice. The results from the longitudinal study are tangible to personal experiences and could be compared to global or long term cognitive mode ('co-creation mode') and with local - short term moral cognition ('coping mode'). The findings demonstrate the students' concern about responsibility and care for ethical space; the determinants fit the 'co-creation mode'. In the same time, the self-imposed limits - within the greatest part of the narratives, appear proper identifications of the target population with integrity' constraints - from the data gives arguments for the 'coping mode'. Both levels of moral cognition are important and explicitly validated in occidental cultures. Starting from this theory this research is distinguished by the exercise of measuring the representation of integrity from a nonoccidental culture with metrics from an occidental culture. The day by day conditions are different, the moral judgments of the respondents are sometimes in conflict, the economic systems do not have the main characteristics, the patterns from Western and non-Western cultures are different. Even the findings are substantial from the vantage point of 'cocreation mode' and 'coping mode', the confronting data suggests that the levels of moral cognition could be extended adding a new level, the 'intuitive mode' (intuitive moral). Maybe the postcolonial morality is not outside of the universal conventions. The text in its' meaning is universal, paradoxically is a telling simple and sophisticated in the same time. The psychological aspects from the Gospel are rich and the ethical significance is multiple. A mature comprehension of the meanings invites us to analyze twofold behaviors, moral disciplines, attitudes, self-expressions, intelligence expressions, socialization mechanisms, relationships or preferences for social interaction, etc.

The relevance of these findings makes evident a retroactive exercise, because integrity [6] is not an inborn skill. The educational communication requires a discussion from the vantage point of moral education. The intent of the debating is dealing with complex relationships between educational communication and moral communication. This question can be reached via plural and conflicting values. Moral thinking, human social behavior, ethical and legal categories of judgment come about when is assumed the making habitual. The recognition of the limits, the adoption of the possibilities and the need of education characters - as aims of moral education - suppose a particular kind of education. It could significantly be argued by the rigidity of totalitarian education. From the perspective of the communication, the Romanian recent history - similarly to past-communist countries - serves different senses of understanding 'morality'. The Marxist writings mystify the moral obligations. In this sense, the term 'morality' does not clarify the rights and behaviors associated, frequently conveys the tendency of fanaticism. It is important to take into account in the research the valid meaning of integrity that is distinguished from the Marx's and Engels's specifically principles, assumptions and norms recognized in the day by day mental. Perhaps this concern was one of the most notoriously difficult parts of the investigation. The religious and secular text provides sense of moral obligations without communist accents and makes intelligible a wider framework of meaning for a moral community alive. Most of the researches on integrity are considered limited because of the gap between moral beliefs and moral conduct; some results are ignored because the participants 'are lying'. The original way to investigate integrity by a practical problem rejects the confusions or false explications of target population. Moral language demonstrates that the moral reasoning investigation fits neutrality in contemporary Romanian climate. The prospects are essential for thinkers in the field and for practice.

In order to establish that responsibility is another key theme of the dilemma, the critical analysis of the data reflects many questions:

1. It is about atypical social behavior?

2. It is about negotiating an 'artificial' responsibility?

3. Are there interferences between natural ethics and oppressed' ethics?

4. Is Martha a 'victim'?

5. How to infer on the relationships between personality and responsibility?

6. How to delineate between public and private responsibility?

This research illustrates the unlearned sophistication of real life, in the context of universal agreement of exact meaning. Perhaps the data from the research could be considered a report on the spiritual, moral, social and cultural development, as a result of reflective approach. Like some other exercises, this paper indicates the importance of relations between values, education, moral development, spiritual intelligence, as between culture, religion, politics and sciences. In terms of teaching religion and philosophy in schools the approach re-creates the question of Pring [9]: "... is this philosophy?" Such dilemma taught at Religion is one of the most influential way to develop moral thinking in Romanian schools. There is an objection concerning the position of Religion in Romanian curriculum despite the fact that after 30 years of past-communism the moral reasoning is seriously affected by the Marx' systematic thinking. The linguistic repertoires of the students refer to the moral brain in sense of conflicting moral values (natural morality and rational morality):"Martha's works materialize; they are 
visible. Both are models." (14). Philosophical objections involve public discourses; the danger of ideological indoctrination still implicit, because of some interests in devaluation of traditional values. The discussion argues the problem of re-gaining the position of the traditional religious education in the Romanian curriculum and certainly to deliberate about its key role in the teaching dilemma. The moral apparatus of religion could engage students to care about their obligations as citizens, in a genuine manner. On the contrary, the cognitive representation about right and wrong - in terms of self-determination, autonomy and social styles - is not philosophically minded (it is closed to indoctrination). Nurture minds are considered a question about values and the dilemmas from the Gospel are 'objective', 'hard moral facts', 'impartial', 'fair-minded'.

Significant implications are expressed in the followings questions:

1. Is education guilty?

2. Are political decisions responsible for the growth of children development?

It is undeniable that it is about influential beliefs by Marxist mechanisms.

The research methodology on moral issues is very complicated, even if the perspectives are focused on psychological, social, cultural or educational determinants. Frequently, the discussions of the outcomes in the fieldwork are multi-paradigmatic. The relations between psychology and religion, between religion and metaphysics, as the relations between sociology and religion evidence continuities, paradoxes and conflicts. This is in contrast to present scientific measurement that relates about identifications and introjections - mechanisms of the personality -, not about intrinsic and extrinsic religiosity. The coding schemes of the narratives were analyzed taking into account the compatibility to a wide range of studies highly structured in terms of objective-subjective, focusedunfocused, explicit-implicit, context dependence and exhaustive-specific. This investigation is an unconventional exercise on integrity, in the sense of adopting the prudential epistemology; the dilemma from the Gospel offers an operational definition of moral principle, both 'hard' and 'soft' facts, a true situation to research, an ordinary meaning of integrity, provides a set of moral standards, exemplary behavior, a successful context of investigation, different logical types of communication, and so on. The difficulties of recording data are equally important because is questionable what a subject says in a questionnaire or in an interview as well as its authentic meanings. It is possible to repeat what he was told. The current territory of honesty is complicated and how the respondents make senses depends on the conceptual framework of the research. The dilemmas correspond to multidimensional conceptual mapping that investigates substantive questions. One of the benefits of this atypical research gives a new start point for future studies: the methodology is sufficiently mature to guide future investigations in other cultures and in other conditions. For example, a dynamic alternative to quantitative or mixed methods might be the use of a variation - emic and ethic perspective on honesty, by ethnomethodology.

Beyond the fact that the communication problems identified two-way connections, the answers are interpreted as reactions to specific communication (communication about values). A mathematical perspective on the issues could guide the inferences; the interplay between the mathematical theory of communication and moral communication produces new meanings on relationships between minimal costs and maximum efficiency. The quantity of moral information determined by each personal experience involved in the task implies taking into account the different outcomes, previously unknown results but equiprobable. May be the mathematical explanation prepared by the 'amount of information' concept of formula

$$
\mathrm{I}=\mathrm{k} \times \log _{10} b
$$

can be represented in the story about Martha and Mary on the basis of statistics. The comparative analysis of the respondents, as a result of processing the universal meaning of the Gospel, denote that they assume the complicated moral judgment in terms of probability: "When I have to choose, I give priority to Martha ... but Mary needs Martha, because faith without work is dead" (5). As a narrative experiment, the relationship between the message length and the quantity of information within deals with an interval closed by uncertainty and certainty as in mathematical theory: 0 probability and 1 probability. Of course, the findings from the narratives evidence a finite probability field: "When I have to choose between Martha and Mary, two roads are opened to me" (26). Both roads signify psychological deals or cultural variables that highlight moral cognition, social cognition, affective cognition according to a predetermined probability. The information entropy within is dependent on the psychological field of the target population. As a result of this, the sophisticated moral cognition could be assessed by the entropy perspective. The formula

$$
\mathrm{I}=\mathrm{H}_{\max }-\mathrm{H},
$$

when it is represented the intensity of information field, $\mathrm{H}_{\max }$ represents maximum entropy and $\mathrm{H}$ represent the entropy field experiment events. The following answer reveals the changing perspective of the findings from the vantage perspective of integrity's entropy: "Firstly, I agreed with both. Then I realized that Martha's deed is temporary"(41). The demonstration is different in case of the dilemma if the approach is focused on the maximum entropy; the outcomes are comparable with mathematical theory - if the maximum entropy is constant, the implications are different. With increasing amounts of information received, therefore, the reduction of informational entropy uncertainty that contains an experiment or a random variable. The huge number of respondents involved in the investigation assures high consistency of the qualitative research. On the other hand, the critical exegesis of the findings delimits in mathematical metrics twofold problems of moral cognition: the individual 
energy spending within the Gospel and the probabilities from the fieldwork. The individual energy information is subject to various probabilities involved in the studied phenomenon. For example, one of the students writes simply: "When I have to choose between Martha's example and Mary's example, in my brain there is a critical process that compares the two examples... there is a balance between the example of Martha and the example of Mary"(30). In order to distinguish the master of moral communication - the costs of every person engaged in such conditions - the multi-causality and the polyphony communication give notable results from the vantage point of knowledge hybridization. The equiprobable outcomes, the degree of uncertainty, the probability that may arise demonstrates that each moral communication message is plausible to be decoded in finite manner. To conclude, the meta-language implies uncertainty because of the features of moral cognition.

The findings could be compared with limits from mathematics. Applying the limits on narratives, the discussion is productive because the scientific rules are similar: the findings are considered a set of real numbers $\mathrm{x}$, in condition of a less than or equal $b$ that can be interpreted in a closed and well-delineated interval $[a, b]$. If the moral judgment is a function (f) defined on the closed and delineated interval [Martha, Mary], the suggestion is to wonder about minimal and maximum values on the interval [Martha, Mary]. In mathematic rationale, where $f$ is continuous on the interval $[a, b]$, then $f(x)$ reaches maximal and minimal values on the interval $[a, b]$. In our approach, where $\mathrm{f}$-moral judgment- is continuous on the interval [Martha, Mary], $\mathrm{f}(\mathrm{x})$ reaches maximal and minimal values on the interval [Martha, Mary]. This process could be schematically described in the following answer: "I weighed the two values - Martha and Mary - and I chose a model value to induce me a model alive. I don't blame Mary's model"(39). The scientific discourse exemplifies the limits theory applied within communication [8]. The moral thinking process is dependent on the moral limitations of legal rules, the philosophy of life, the consciousness, the attitudes, the social responsibility, the natural law, and produces the meaning of meaning.

It is possible that the applications of the probability in moral communication having a 'research transfer' as start point - the communication concept from the quants theories is transferred in moral communication. In the same time it is about a hybridization between moral education, probabilities, quants theories, linguistic and semiotics, communication sciences and ethics. There are various arguments: different forms of knowledge are characterized by heteroclites concepts. For mathematics and logics are essentials inferences, for physics are important relationships cause and effect, for morals - good and wrong, for philosophy - sense, for religion - respect. The understanding of moral communication require taking into account the certainty and the uncertainty of meaning based on contradictions and noncontradictions, syllogism, observation, situation control, sensibility. The moral meanings are dependent by values day by day - altruism vs. moral egoism, good vs. bad, legal rights vs. moral rights, own values vs. common values, honesty vs. corruption. The moral facts have not meaning: the values give the meaning of moral facts.

In terms of knowledge, moral communication implies factual knowledge, 'how' knowledge, knowledge by acquaintance. According to Hirst [10], the moral knowledge could be delimited as following:

1. knowledge a moral person,

2. knowledge how are we morals,

3. knowledge of factual morals,

4. knowledge the causes of corruption,

5. knowledge of who is a moral example,

6. knowledge of integrity,

7. knowledge the way of moral eccentricity,

8. knowledge of how to be faithful.

In science are essential the arguments [11], not the opinions $[12,13,14,15]$; in investigation of integrity the affirmation 'I know' refers to a probable camp. The consequence is strong: the truth condition is function by different probabilities that are involved in the phenomenon because the relationship between moral argue, moral beliefs, mental states is ambiguous. The discourses on 'moral knowledge' are discontinuous: it is about a moral agent (a personal view), the evaluative sense of the term moral (in sense of 'my moral'), the descriptive part of notion moral (connected to the various fields - scientific, artistic, religious, politic, etc.). We consider that is not a moral judgment, there are variations of moral judgment and the moral rationale is an 'unsolved solution'.

\section{Conclusion}

All the findings and the discussions lead to general remarks and suggestions. The paper gives an overview on the conceptual structure of the law analyzing 'moral law' and 'legal law'. There are no similar approaches in the fieldwork in order to compare the results, but the research could be replicated in various cultures. Even the common law varies from country to other, the meanings of legal concepts empower moral rights. The results of the investigation are rich from the vantage point of moral cognition, personality, linguistic and semiotics, judicial and moral brain. The inferences reflect the 'moral' version of responsibility as a deliberate human choice. The qualitative research on integrity had a substantial contribution in investigating moral reasoning of persons as moral agents. The examination illustrates the incompatibility between moral rights and legal rights as bases for transnational law and adds new psychologically insights for future policy science (moral cognition).

To conclude, the findings prove that the multiculturalism implies taking into account the social, biological and cultural situatedness of the indigenous people. The assumption was validated: a clear meaning of the moral task sustains basic psychology. Critical reflection shows that the new initiatives and research strategies in the fieldwork are imperatives in 
close relation to producing new insights in human psychology. Speaking intra-psychically the communication exercise illustrates a culture of moral principles. The integrity test in the formula of a moral communication pretext can be adapted to suit a wide variety of research situations and various purposes and assure high standards' transparency. For example, the standardized structured interview schedule allows important characteristics of the candidate to the magistrates' profession. There are various advantages for empirical work, taking into account the transferability and the generalizability of the findings. It is about conversational openings: the scientific investigation requires responsibility: it is no doubt that Mary needs Martha because by Martha, Mary is proud too" as in Desert Fathers from Sinai.

Following questions could guide the future studies in the fieldwork:

1. Is moral communication a 'border concept' or could be considered a concept diffused from a science to another?

2. Could be interpreted moral communication as a currency between the sciences?

3. Is moral communication a bridge assuring the intellectual migration between knowledge?

4. How to impose global standards on honesty when the moral judgment is so dependent on faith?

5. How to infer on moral cognition in terms of laboratory paradigm?

\section{References}

[1] M. Argyle (2000). Psychology and Religion. An Introduction. London \& New York: Routledge.

[2] J. Bruner (2009). Culture of education. Harvard: Harvard University Press.

[3] H. Gardner (2009). Multiple Approaches to Understanding. in Illeris (ed.), Contemporary Theories of Learning. Learning Theorists... In Their Own Words (pp. 106-115). New York: Routledge.
[4] V. Trif (2020). Analyzing Paradigms Used in Education and Educational Psychology. New York: IGI Global.

[5] M. Kaptein (2005). The Six Principles of Managing with Integrity: Practical Guide for Leaders. London: Spiro, (pp. 36)

[6] V. Trif (2014). A Qualitative Perspective on Integrity. In Journal of Education, Psychology and Social Sciences, volume 2 issue 1 , (pp. 38-43).

[7] M. Delgado., E. Phelps, \& T. Robbins (2011). Decision Making, Affect, and Learning. Oxford: Oxford University Press.

[8] A. Sneddon (2011). Like-Minded. Externalism and Moral Psychology. Cambridge: The MIT Press.

[9] R. Pring, R. (2008). Philosophy and Moral Education. In M. Hand \& C. Winstanley (eds.), Philosophy In Schools (pp. 1840). London and New York: Continuum.

[10] P. Hirst (1974). Knowledge and the Curriculum: a Collection of Philosophical Papers. London: Routledge.

[11] L. Tauginiene, E. Butkeviciene, K., Vohland et al. (2020). Citizen Science in the Social Sciences and Humanities: the Power of Interdisciplinarity. Palgrave Communications 6, 89. https://doi.org/10.1057/s41599-020-0471-y.

[12] B. Enke (2019). Kinship, Cooperation, and the Evolution of Moral Systems. The Quarterly Journal of Economics 134, 2. https://doi.org/10.1093/qje/qiz001 (pages 953-1019)

[13] J. Amstrong, R. Friesdorf, P. Conway. (2019). Clarifying Gender Differences in Moral Dilemma Judgments: The Complementary Roles of Harm Aversion and Action Aversion. Social Psychological and Personality Science 10, 3 Doi: 10.1177/1948550618755873 (pages 353-363).

[14] R. O., Salvador (2019). Reexamining the "Discussion" in the Moral Dilemma Discussion. Journal of Business Ethics 156. https://doi.org/10.1007/s10551-017-3626-z (pages 241-256).

[15] I., Patil, M. M., Zucchelli, W., Campbell, S., Fornasier, F., Calo et al. (2020). Resoning Supports Utilitarian Resolution to Moral Dilemmas across Diverse Measures. Journal of Personality and Social Psychology. https://doi.org/10.1037/pspp0000281. 